\title{
Characterisation of a cell wall-anchored protein of Staphylococcus saprophyticus associated with linoleic acid resistance
}

\author{
Nathan P King ${ }^{1}$, Türkan Sakinç ${ }^{2,4}$, Nouri L Ben Zakour ${ }^{1}$, Makrina Totsika', Begoña Heras ${ }^{3}$, Pavla Simerska ${ }^{1}$,
} Mark Shepherd ${ }^{1,5}$, Sören G Gatermann², Scott A Beatson ${ }^{1}$ and Mark A Schembri ${ }^{{ }^{*}}$

\begin{abstract}
Background: The Gram-positive bacterium Staphylococcus saprophyticus is the second most frequent causative agent of community-acquired urinary tract infections (UTI), accounting for up to $20 \%$ of cases. A common feature of staphylococci is colonisation of the human skin. This involves survival against innate immune defenses including antibacterial unsaturated free fatty acids such as linoleic acid which act by disrupting bacterial cell membranes. Indeed, S. saprophyticus UTI is usually preceded by perineal skin colonisation.

Results: In this study we identified a previously undescribed $73.5 \mathrm{kDa}$ cell wall-anchored protein of $\mathrm{S}$.

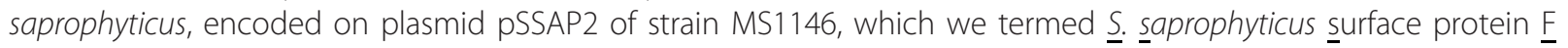
(SssF). The sssF gene is highly prevalent in S. saprophyticus clinical isolates and we demonstrate that the SssF protein is expressed at the cell surface. However, unlike all other characterised cell wall-anchored proteins of $S$. saprophyticus, we were unable to demonstrate a role for SssF in adhesion. SssF shares moderate sequence identity to a surface protein of Staphylococcus aureus (SasF) recently shown to be an important mediator of linoleic acid resistance. Using a heterologous complementation approach in a S. aureus sasF null genetic background, we demonstrate that SssF is associated with resistance to linoleic acid. We also show that S. saprophyticus strains lacking SSSF are more sensitive to linoleic acid than those that possess it. Every staphylococcal genome sequenced to date encodes SssF and SasF homologues. Proteins in this family share similar predicted secondary structures consisting almost exclusively of $\alpha$-helices in a probable coiled-coil formation.

Conclusions: Our data indicate that SSSF is a newly described and highly prevalent surface-localised protein of S. saprophyticus that contributes to resistance against the antibacterial effects of linoleic acid. SssF is a member of a protein family widely disseminated throughout the staphylococci.
\end{abstract}

\section{Background}

Urinary tract infections (UTIs) are a universal source of human morbidity, with millions of cystitis and pyelonephritis episodes reported annually [1]. An estimated 40$50 \%$ of all women will experience at least one UTI in their lifetime, and one in three women will have had at least one clinically diagnosed UTI by the age of 24 [2]. Direct health care costs due to UTI exceed $\$ 1$ billion each year in the USA alone [2]. Staphylococcus saprophyticus, a

\footnotetext{
* Correspondence: m.schembri@uq.edu.au

${ }^{1}$ Australian Infectious Diseases Research Centre, School of Chemistry and Molecular Biosciences, The University of Queensland, Brisbane, QLD 4072, Australia

Full list of author information is available at the end of the article
}

coagulase-negative staphylococcus, is the second most common causative agent of community-acquired urinary tract infection after Escherichia coli [3], and is responsible for up to $20 \%$ of cases. S. saprophyticus is of particular significance to sexually active young women, accounting for over $40 \%$ of UTI in this demographic [4]. S. saprophyticus UTI symptoms mirror those of E. coli [5] and recurrence is common, affecting $10-15 \%$ of infected women [6].

Three cell wall-anchored proteins, featuring a conserved characteristic C-terminal LPXTG motif, have previously been identified in S. saprophyticus. These proteins (i.e. SdrI, UafA and UafB) are all involved in adhesion [7-9], a crucial first step in the colonisation process. S. saprophyticus also possesses non-covalently surface-associated Aas $[10,11]$

\section{Biomed Central}


and Ssp [12] proteins that are implicated in virulence. Other than surface proteins, S. saprophyticus produces abundant urease which contributes to its ability to grow in urine [13]. Other putative virulence factors include cell surface hydrophobicity [14], slime [15] and D-serine deaminase [16].

Apart from rare complications, S. saprophyticus is only known to infect the urinary system [17-19]. The primary niches of this organism are in the human gastrointestinal and genitourinary tracts $[4,20]$. S. saprophyticus UTI is often preceded by colonisation of the perineal area; thus it can survive despite the innate immune defences of the skin. In this study, we have identified a previously undescribed LPXTG motif-containing cell wall-anchored protein of S. saprophyticus, termed SssF. The sssF gene is plasmid-encoded in S. saprophyticus strains ATCC 15305 and MS1146 and is highly prevalent in clinical isolates. We show that SssF belongs to a family of proteins conserved among staphylococcal species and contributes to survival against the staphylocidal free fatty acid linoleic acid - a component of the human innate immune defence system.

\section{Results}

\section{Analysis of plasmid pSSAP2}

S. saprophyticus strain MS1146, a clinical UTI isolate, has been described previously [7]. Its genome contains three plasmids - pSSAP1, pSSAP2 and pSSAP3. Sequence analysis of the $36907 \mathrm{bp}$ pSSAP2 plasmid revealed the presence of 35 predicted protein-coding genes, six pseudogenes and a mean $\mathrm{G}+\mathrm{C}$ content of $29.9 \%$ (Figure 1 and Additional file 1: Table S1). Like other staphylococcal plasmids previously described, pSSAP2 has a mosaic structure with evidence of multiple insertions and deletions of discrete sequence blocks.

Plasmid pSSAP2 contains the repA gene and an approximately $17 \mathrm{~kb}$ region (from position 4124 to 21 247) which share $96 \%$ and $97-99 \%$ nucleotide identity, respectively, with the chromosome of $S$. saprophyticus ATCC 15305 (Figure 1). A large proportion of the proteins encoded in this region are of unknown function or hypothetical, with the exception of a putative permease and several analogues of enzymes of the ribulose monophosphate pathway (Additional file 1: Table S1). Of note, the corresponding region in S. saprophyticus ATCC 15305 is longer $(26 \mathrm{~kb})$ and contains an arsenic resistance operon $\operatorname{ars} R B C$ and a putative lipase, both absent from pSSAP2. This region is also framed by two copies of the IS element IS431, which is frequently involved in the recombination-mediated integration of transposons and plasmids in methicillin-resistant S. aureus (MRSA) chromosomes [21,22]. Therefore, this region is likely to be an integrative plasmid of strain ATCC 15305; positioned upstream is a truncated integrase (SSP1642), for which an intact copy can be found in the S. saprophyticus MS1146 chromosome (Figure 1).

Another region of pSSAP2, ranging from position 21 529 to 33235 , shares $~ 99 \%$ nucleotide identity with plasmid pSSP1, which was originally described from S. saprophyticus ATCC 15305 [8]. The most notable feature of this region is the presence of a gene encoding for a LPXTG domain containing protein that we have designated $\operatorname{ss} F$ (see below).

\section{Sequence analysis of SssF staphylococcal homologues}

The S. saprophyticus MS1146 sssF gene is 1962 bp in length and the full-length translated SssF (S. saprophyticus surface protein $\mathrm{F}$ ) protein contains 654 residues with a predicted molecular mass of $73.5 \mathrm{kDa}$ (Figure 2A). SssF contains a predicted signal peptide of 45 residues (SignalP) [23] and an LPDTG anchor motif at the $\mathrm{C}$ terminus (Figure 2A), involved with covalent attachment of the mature protein to the cell wall. No conserved functional protein domains were detected, except for a possible albumin-binding GA module (Pfam PF01468, residues 58-109, E-value = 0.00039).

Sequence searches using the SssF amino acid sequence revealed similar proteins in other staphylococci. As expected, the SssF homologue encoded by pSSP1 in S. saprophyticus ATCC 15305 is near-identical at the protein level with only seven amino acid substitutions. Of note, every other sequenced staphylococcal genome contains an sssF-like gene, all chromosomally located except in S. saprophyticus (Additional file 2: Figure S1). Multiple alignment of the $\mathrm{C}$-terminal regions (corresponding to the C-terminal 402 residues of SssF sequence) of one representative SssF-like protein from each sequenced staphylococcal species demonstrates there is variation from blocks of conserved and similar residues to regions of less similar sequence. This showed an overall protein identity ranging from 30.3-47.6\%, versus Staphylococcus pseudintermedius HKU10-03 and Staphylococcus carnosus TM300, respectively, and an average amino acid identity of approximately $37 \%$ with the remaining SssF-like proteins. In terms of protein sequence similarity, these values range from $41.7 \%$ (S. pseudintermedius HKU10-03) to $84.4 \%$ (S. carnosus TM300). The N-terminal sequences are considerably more divergent.

All SssF-like proteins have a predicted signal peptide of between 35 and 45 residues, according to SignalP predictions. It is noted that the annotated Staphylococcus haemolyticus JCSC1435 SssF-like protein has an incorrectly called start codon, artifactually truncating the signal peptide sequence. All of the SssF-like proteins have a Cterminal sortase motif, implying cell surface localisation. Of the ten illustrated in Additional file 2: Figure S1, four have the canonical LPXTG motif, five have an alanine 


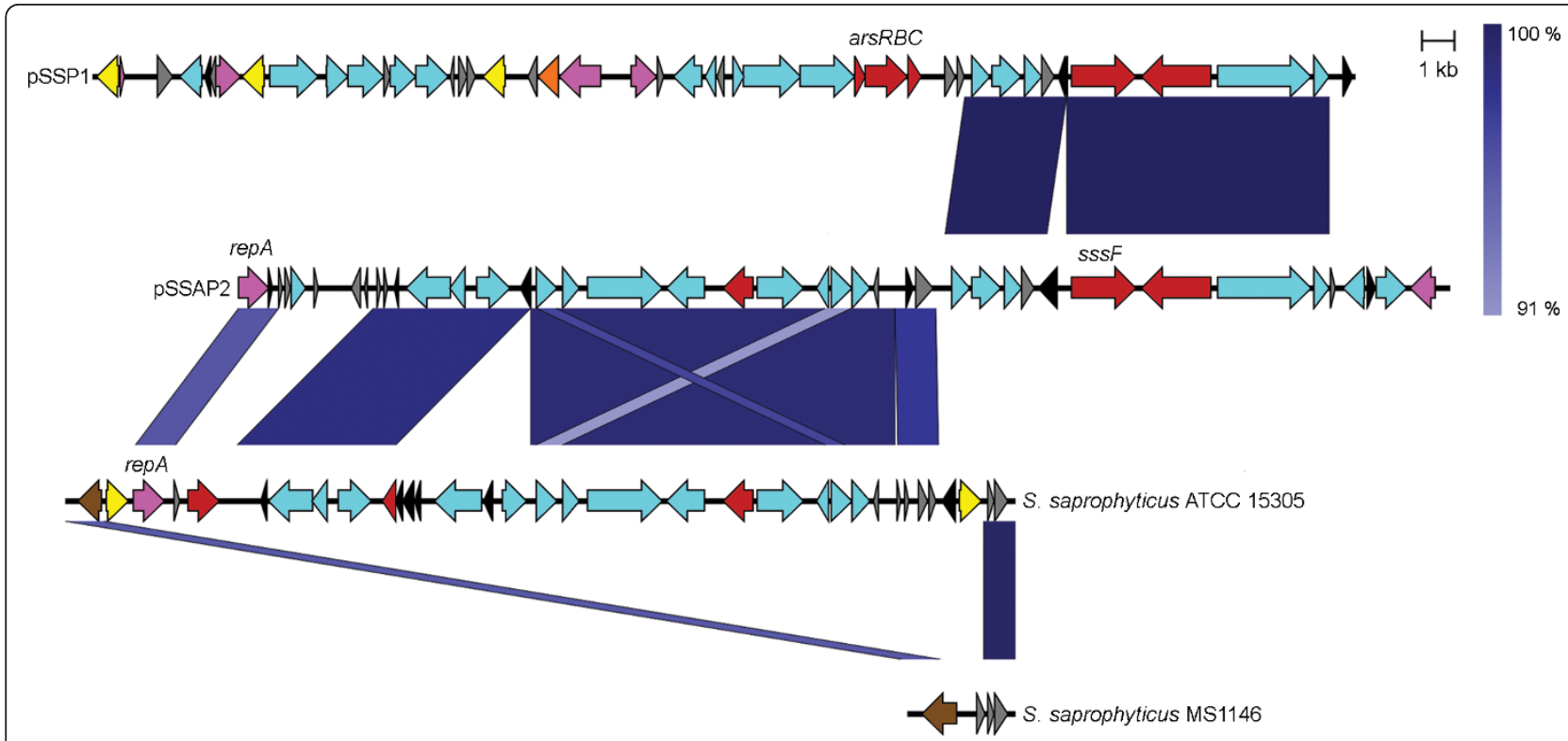

Figure 1 Structure of the S. saprophyticus MS1146 plasmid pSSAP2 compared to the S. saprophyticus ATCC 15305 plasmid pSSP1, and the chromosomes of S. saprophyticus ATCC 15305 and S. saprophyticus MS1146. Arrows represent CDS coloured according to their predicted function: no specific function (light blue); replication (pink); transposase for IS431 (yellow); other transposase (orange); integrase (brown); virulence-related (red); hypothetical protein (grey); and pseudogenes (black). Similarity regions between sequences are coloured in a gradient of blue, reflecting the percentage of nucleotide identity ranging from 91 to 100\%, as illustrated on the scale on the top right of the figure.

residue in the fourth position, and the Staphylococcus lugdunensis protein has a serine in this position.

\section{Structural prediction of SssF}

Secondary structure predictions using PSI-PRED [24] indicate that SssF contains long, almost uninterrupted segments of $\alpha$-helices (Figure $2 \mathrm{~B}$ ), which are likely to wrap around each other forming a rope-like coiled-coil structure. In order to predict its three-dimensional fold we carried out a fold-recognition analysis of SssF sequence using Phyre [25] (Protein Homology/AnalogY Recognition Engine). This server allows a pairwise alignment of the SssF sequence to a library of known protein structures available from the Structural Classification of Proteins (SCOP) [26] and the Protein Data Bank (PDB) [27] databases and generates preliminary models of the protein by mapping the sequence onto the atomic coordinates of different templates. Although SssF shares very low sequence identity with proteins in the PDB (range from 5-9\%), this analysis identified several structural homologues of SssF with a confidence level of $100 \%$. All the structures identified as likely analogues of SssF correspond to proteins that have a coiled-coil fold, including various types of the filamentous proteins such as tropomyosin [28] (PDB code: 1C1G) or alpha-actinin [29] (PDB code $1 \mathrm{HCI}$ ) (Figure $2 \mathrm{C}$ ), strongly suggesting that this protein shares a similar three-dimensional structure. Each of the SssF-like proteins (complete mature forms) of the other ten staphylococcal species indicated in Additional file 2: Figure $\mathrm{S} 1$ is also predicted to almost exclusively consist of $\alpha$-helical coiled-coils with the same Phyre-predicted structural analogues as SssF (data not shown).

\section{The sssF gene is highly prevalent in S. saprophyticus}

To assess the prevalence of sssF in S. saprophyticus we used PCR to screen our collections of clinical isolates originating from Australia, Germany and the USA. The sssF gene was detected in $84.6 \%$ (55/65) of Australian isolates, $90.9 \%(10 / 11)$ of American isolates and 88.3\% $(53 / 60)$ of German isolates.

\section{SssF is expressed at the S. saprophyticus cell surface}

In order to study the cellular localisation and function of the SssF protein, we generated an isogenic S. saprophyticus MS1146 sssF mutant (MS1146sssF) by insertional inactivation with a group II intron using the TargeTron system. We then complemented the sss $F$ mutation by the introduction of a pPS44 staphylococcal vector containing the cloned sssF gene, to create MS1146sssF(pSssF). Western blot analysis of whole-cell lysates from S. saprophyticus MS1146, MS1146sss $F$ and MS1146sssF(pSssF) using rabbit polyclonal anti-SssF serum raised against a recombinant truncated SssF protein, demonstrated expression of SssF in MS1146 but not MS1146sssF. Complementation of $s s s F$ restored SssF expression in MS1146sssF(pSssF) (Figure 3A). The 


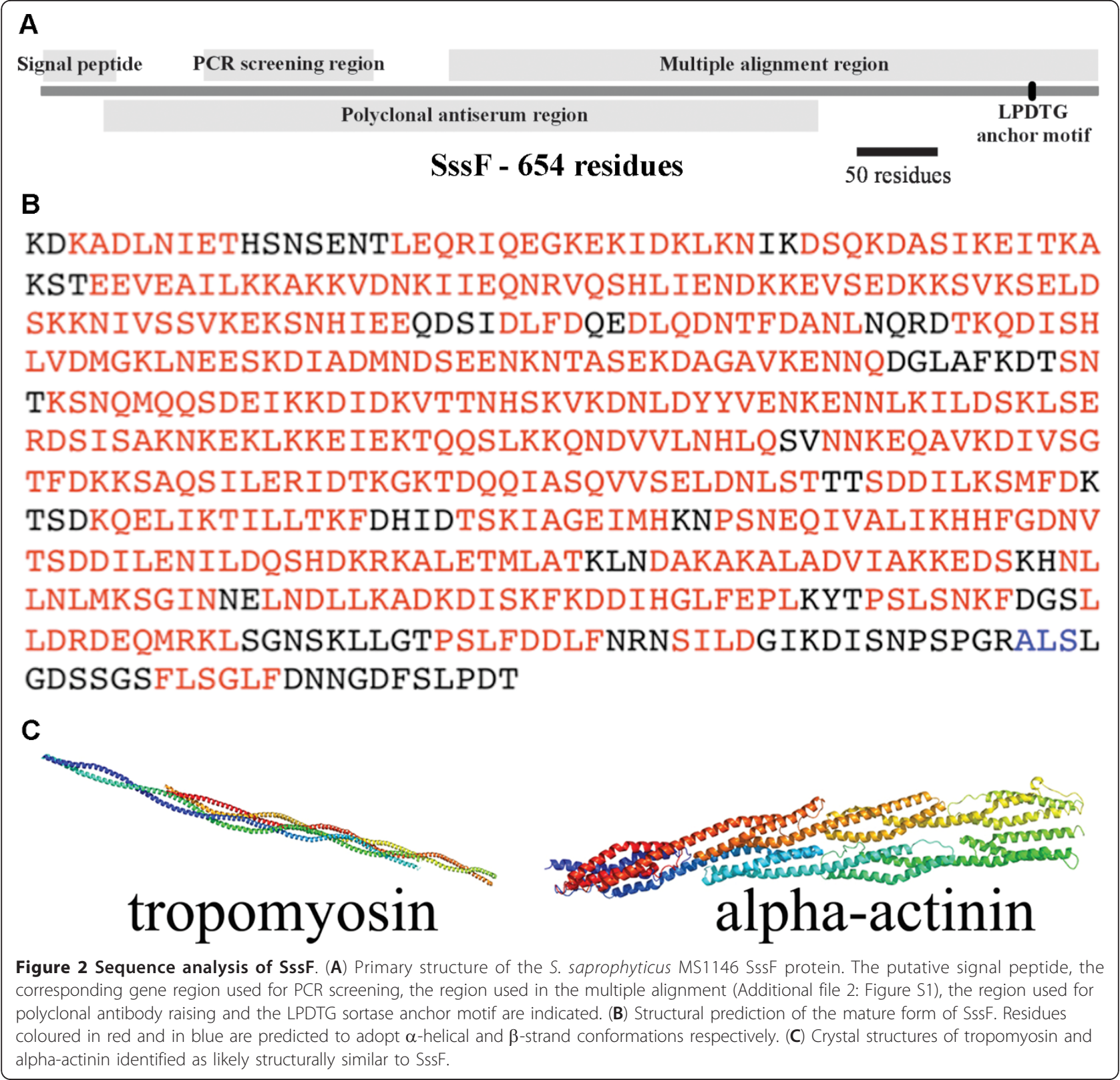

anti-SssF serum was used in conjunction with immunogold labeling and electron microscopy to demonstrate localisation of the SssF protein at the cell surface. MS1146 and MS1146sssF(pSssF) exhibited abundant gold labeling whereas MS1146sssF was devoid of labeling (Figure 3B).

SssF does not mediate adhesion to uroepithelial cells or colonisation of the mouse bladder

Initial investigations into the function of SssF found no evidence of adhesion (to T24 and 5637 human bladder carcinoma cells [American Type Culture Collection; ATCC], exfoliated human urothelial cells or a wide range of ECM and other molecules, including human serum albumin), invasion of 5637 bladder cells, cell surface hydrophobicity modulation, biofilm formation or serum resistance that could be attributable to SssF (data not shown). Strain MS1146 and derivatives colonised the mouse bladder in similar numbers in a mouse model of UTI (4.8-5.8 $\times 10^{6}$ c.f.u. per $0.1 \mathrm{~g}$ bladder tissue), indicating that SssF does not contribute to colonisation in this infection model.

S. saprophyticus strains containing the sssF gene are more resistant to linoleic acid than those lacking $s s s F$

The above results prompted us to analyse the sequences of the family of SssF-like proteins to predict a function 


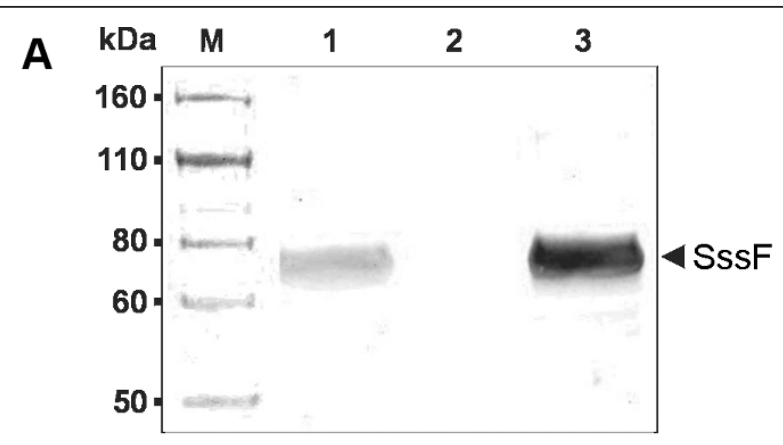

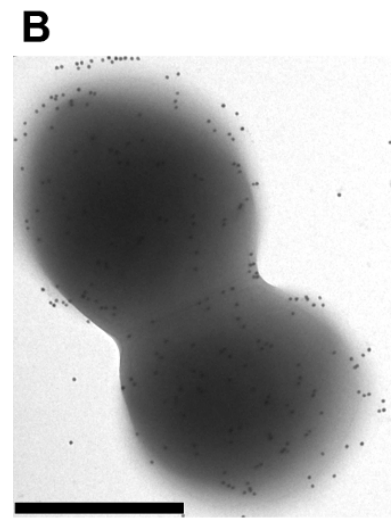

MS1146

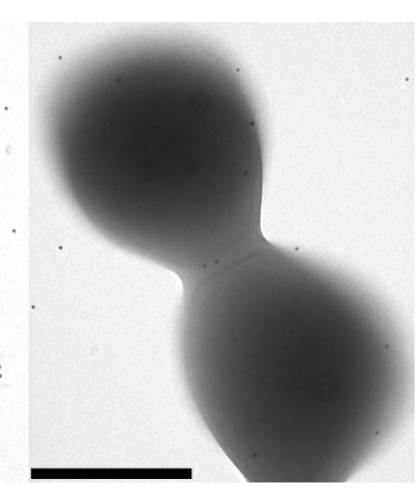

MS1146sssF

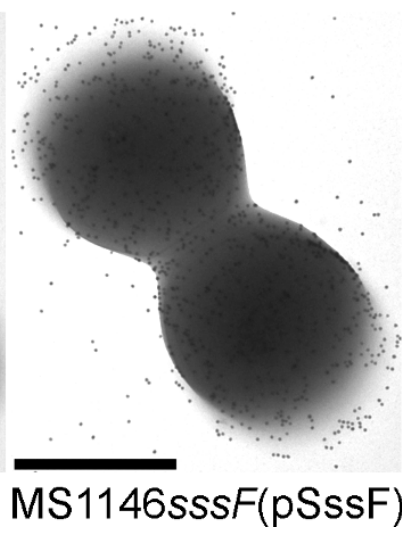

MS1146sssF(pSssF)

Figure 3 Expression of SssF. (A) Western blot analysis of whole-cell lysates prepared from S. saprophyticus MS1146, MS1146sssF and MS1146sssF (pSssF) using a polyclonal antiserum directed against SssF. Lanes: M, Novex Sharp Pre-stained protein marker (Invitrogen); 1, MS1146; 2 , MS1146sssF; 3, MS1146sssF(pSssF). The position of SssF is indicated. Expression of SssF was detected in wild-type S. saprophyticus strain MS1146 and the sSSF complemented strain but not in the isogenic ssSF mutant. (B) Immunogold TEM of S. saprophyticus MS1146, MS1146sssF and MS1146sssF(pSssF). Expression of SssF at the cell surface of S. saprophyticus MS1146 was demonstrated by abundant labeling with SssF-gold particles. In contrast, the ssSF isogenic knockout mutant was devoid of gold labeling. Complementation of the sssF mutation restored and enhanced surface expression of SssF. Bars, $500 \mathrm{~nm}$.

for SssF. The staphylococcal SssF-like proteins are all hypothetical proteins of unknown function except for SssF, which contributes to resistance of $S$. aureus to linoleic acid [30]. The mechanism of this phenotype remains unexplored. To determine whether SssF had a similar phenotype to the $S$. aureus SasF protein, linoleic acid survival assays were performed with $S$. saprophyticus MS1146 wild-type, MS1146sssF and MS1146sssF (pSssF) strains. No differences in survival among the strains were observed (data not shown). Following the lack of an observable phenotype for SssF in S. saprophyticus MS1146, we modified the linoleic acid emulsion assay to examine the survival of S. saprophyticus isolates that contain and do not contain the sssF gene in the presence of $0.85 \mathrm{M} \mathrm{NaCl}$. Under these conditions, we observed a 30 -fold difference in survival between the $s s s F^{+}$and $s s F^{-}$strains $(P=0.008$; Figure 4$)$. Using this modified protocol, we still observed no difference between the $S$. saprophyticus MS1146 wild-type and sssF mutant at linoleic acid concentrations of up to $25 \mathrm{mM}$ (data not shown).

\section{SssF is associated with resistance to linoleic acid}

Survival assays were carried out with a S. aureus SH1000 genetic background, with the aim of determining if SssF could restore linoleic acid resistance of a $S$. aureus SH1000sasF knockout mutant (Figure 5). In agreement with a previous study [30], mutation of $s a s F$ in $S$. aureus SH1000 resulted in enhanced sensitivity to linoleic acid and this effect could be complemented by the introduction of a sasF-containing plasmid [SH1000sasF(pSKSasF)]. When the sssF gene from S. saprophyticus MS1146 was introduced into $S$. aureus SH1000sasF, resistance to linoleic acid was also restored, demonstrating that SssF contributes to the survival of $S$. aureus in the presence of linoleic acid.

\section{Discussion and conclusion}

S. saprophyticus is a major cause of community-acquired UTI in young women. Knowledge of the virulence mechanisms of S. saprophyticus has advanced in recent years, particularly with the acquisition and analysis of whole genome sequence data. The majority of acknowledged virulence 


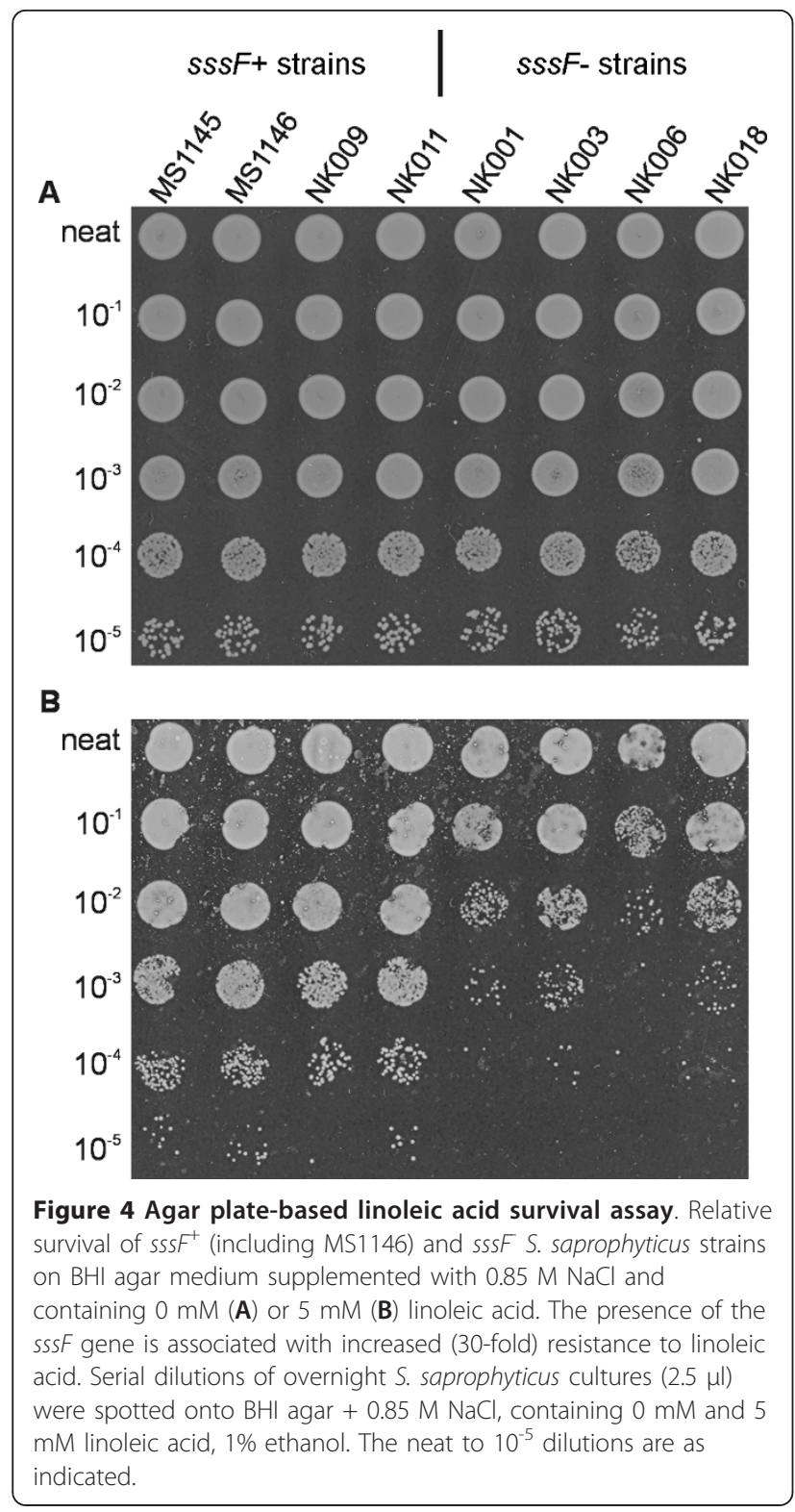

factors of S. saprophyticus are proteins tethered to the cell surface, which with the exception of the Ssp lipase [12], are all involved in adhesion: Aas is an autolysin that also binds to fibronectin [10]; UafA adheres to uroepithelial cells via an unidentified ligand [8]; SdrI binds to collagen I and fibronectin $[9,31]$ and UafB binds to fibronectin, fibrinogen and urothelial cells [7]. Here we have identified another cell wall-anchored protein produced by $S$. saprophyticus that we have termed SssF - the sixth surface protein described for this species.

The $s s F$ gene was identified in the sequence of the pSSAP2 plasmid of S. saprophyticus MS1146 due to the presence of the canonical LPXTG sortase motif in the translated protein sequence. A copy of the $s s s F$ gene is also located on the pSSP1 plasmid of S. saprophyticus

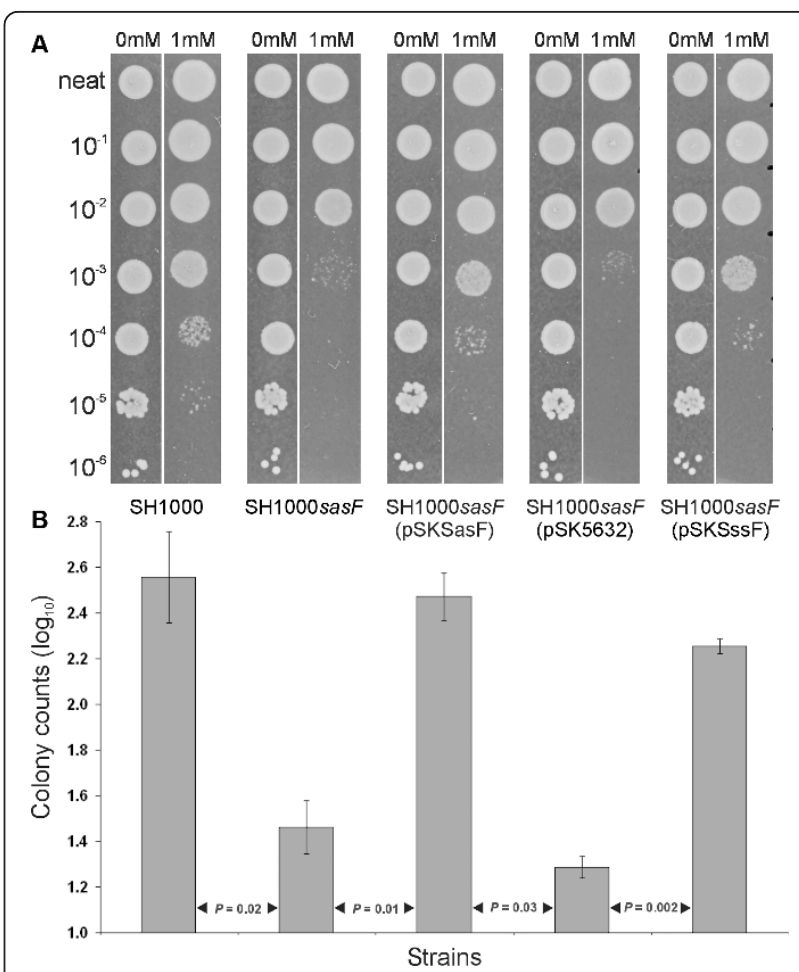

Figure 5 SssF activity is detected in a S. aureus heterologous complementation approach. (A) Relative survival of S. aureus SH1000 wild-type, SH1000sasF isogenic mutant and sasF, sssF and vector only complemented strains on agar medium containing 1 $\mathrm{mM}$ linoleic acid. Heterologous complementation of the $S$. aureus SH1000 sasF mutant with the SSSF gene from S. saprophyticus MS1146 restores survival in these conditions. Serial dilutions of overnight $\mathrm{S}$. aureus cultures $(2.5 \mu \mathrm{l})$ were spotted onto $\mathrm{BH}$ agar, $\mathrm{pH}$ 6.0, containing $0 \mathrm{mM}$ and $1 \mathrm{mM}$ linoleic acid, $1 \%$ ethanol. The neat to $10^{-6}$ dilutions are as indicated. Shown are representative images from one of multiple experiments. (B) Graph showing the relative survival of S. aureus $\mathrm{SH} 1000$ and $\mathrm{SH} 1000$ derivates using data from Figure 5A. Colonies were counted after overnight incubation. Error bars represent \pm SEM. Results from multiple experiments were analysed with Student's $t$ test.

ATCC 15305 (99\% nucleotide identity; Figure 1), but it was not acknowledged as encoding an LPXTG motifcontaining protein [8]. We recently characterised another plasmid-coded LPXTG motif-containing protein of S. saprophyticus MS1146, UafB, as an adhesin [7]. We first sought to investigate whether SssF was another adhesin, since a considerable proportion of characterised Gram-positive covalently surface anchored proteins have adhesive functions [32], including every other known S. saprophyticus LPXTG motif-containing protein. No evidence of an adhesion phenotype for SssF was detected.

SssF protein sequence searches with the BLAST database provided an output of uncharacterised staphylococcal proteins with a maximum $39 \%$ amino acid identity to SssF across the entire protein sequence, mostly annotated as 
hypothetical cell wall-anchored proteins. In contrast to S. saprophyticus, the genes encoding these SssF-like proteins are located on the chromosome, rather than on a plasmid, in every other sequenced staphylococcal species. Some of these staphylococcal SssF-like proteins contain atypical sortase motifs. At this stage it is not known whether all of these proteins are sorted to the cell surface efficiently, but SasF has been shown to be associated with the cell wall of $S$. aureus 8325-4 even with the non-classical LPKAG sortase motif [33]. There was a distinct lack of phenotypic data for these SssF-like proteins until a role for SasF was recently uncovered. Kenny et al. [30] observed that sasF was the most upregulated gene in $S$. aureus MRSA252 microarray and qRT-PCR experiments upon challenge with linoleic acid. The protective function of SasF was apparent when examined in a linoleic acid emulsion agar plate-based bacterial survival assay. Our hypothesis focused on the possibility that SssF possessed a similar function to SasF, but no linoleic acid resistance phenotype for SssF was observed in the S. saprophyticus MS1146 genetic background. Using the linoleic acid emulsion agar plate bacterial survival assay in the presence $0.85 \mathrm{M} \mathrm{NaCl}$, we observed a higher survival amongst $S$. saprophyticus strains that harbour the $s s s F$ gene than those that lack $s s s F$. We then successfully expressed SssF heterologously in a S. aureus SH1000sasF host and demonstrated restored resistance to linoleic acid. We found S. saprophyticus MS1146 to be intrinsically more resistant to linoleic acid than $S$. aureus SH1000. This remains to be explored but could be due to a number of species/strain specific factors including the action of redundant $S$. saprophyticus MS1146 resistance mechanisms or variations in surface components such as capsule or teichoic acids.

We found that the survival of S. aureus SH1000 and its derivatives was markedly increased in the presence of linoleic acid at pH 6.0 compared to $\mathrm{pH}$ 7.4. This result is consistent with previous studies of the staphylococcal fatty acid modifying enzyme (FAME), an unidentified but partially characterised protein secreted by most staphylococci which detoxifies free fatty acids by esterifying them to an alcohol $[34,35]$. The FAME of S. aureus and S. epidermidis demonstrate optimal activity at $\mathrm{pH} 6.0$, and have little activity at $\mathrm{pH} 7.4[35,36]$. This is congruent with human skin having a slightly acidic $\mathrm{pH}$ of 5.5-6 [37]. RP-HPLC experiments using linoleic acid and crude protein extracts demonstrated that SssF activity is distinct from FAME activity (data not shown). Other antimicrobial fatty acids such as sapienic acid have yet to be examined as substrates for SssF or SasF. We hypothesise that some or all of the other uncharacterised SssF-like proteins exhibit fatty acid resistance activity, but this remains to be demonstrated experimentally.

There are precedents for bacterial surface structures that provide protection against bactericidal free fatty acids. Gram-positive bacterial cell wall teichoic acids provide protection against free fatty acid mediated killing of $S$. aureus [38]. The IsdA protein of $S$. aureus reduces bacterial hydrophobicity when expressed at the cell surface under the cue of iron starvation to resist fatty acid membrane attack and also promotes fatty acid resistance of S. aureus in a volunteer human skin survival model [39]. Our studies however found that expression of SssF does not influence cell surface hydrophobicity of S. saprophyticus, and this corresponds with matching data for SasF and S. aureus [30].

No conserved motifs that might predict the functional residues of SssF-like proteins were identified. The observation that the SssF-like proteins are structurally related to myosin is noteworthy, especially in light of the recent characterisation of myosin cross-reactive antigens of Streptococcus pyogenes and Bifidobacterium breve as fatty acid hydratases $[40,41]$. These enzymes act to detoxify unsaturated free fatty acids, including linoleic acid. Homologous proteins with modest primary sequence identity but similar tertiary structures are acknowledged in both bacterial [42] and mammalian [43] lipid-binding protein families. It is possible that conserved tertiary protein structure between SssF-like proteins contributes to their function.

S. saprophyticus is a uropathogen, but SssF is unlikely to have evolved to facilitate survival in the urinary tract. A common trait of staphylococci is skin colonisation. Staphylocidal free fatty acids (especially unsaturated) are present on human skin [44] and are also active in staphylococcal abscesses [45]. Furthermore, linoleic acid is one of the most abundant polyunsaturated fatty acids on human skin [46], and is also present in vaginal secretions [47]. SssF may be an important determinant for survival of $S$. saprophyticus in the events preceding urethral entry in community-acquired UTI - colonisation of perineal and periurethral tissue. This would account for the absence of SssF involvement in the mouse model of UTI, in which the inocula are delivered directly into the bladder.

The location of $s s s F$ on a plasmid in both sequenced $S$. saprophyticus strains is intriguing, particularly as every other staphylococcal SssF-like protein is chromosomally encoded. It has been observed that many genes that are located on plasmids encode for traits which have extracellular functions [48], and $s s s F$ falls into this category. Furthermore, plasmid genes have often been noted to confer selective advantage to the bacteria in some environmental niches but not others [49]. Every pathogenic staphylococcal species known to carry a chromosomal sssF-like gene is known to commensally inhabit the skin, and this can be considered their main niche. S. saprophyticus, on the other hand, primarily resides in the genitourinary and gastrointestinal tracts $[4,20]$. It is feasible that since human skin is not the major habitat of $S$. saprophyticus, sssF has been 
retained as an accessory gene required for survival on the skin during non-UTI periods. Nonetheless, it may still be the case that $s s s F$ is found on the chromosome of some $S$. saprophyticus strains.

SssF represents the fourth LPXTG motif-containing protein described in S. saprophyticus. We present here evidence that the S. saprophyticus SssF protein has a role in the protection against free fatty acid mediated killing, and that it is a member of a newly identified protein family broadly distributed throughout the Staphylococcus genus.

\section{Materials and methods}

\section{Bacterial strains and plasmids}

The bacterial strains and plasmids used in this study are listed in Table 1. The clinical S. saprophyticus isolate collection used in this study is as previously described [7]. In addition, 60 clinical isolates from Germany were also tested. S. saprophyticus ATCC 15305 was described previously [8]. Staphylococcal strains were cultured in/ on Brain Heart Infusion (BHI) broth/agar (Oxoid) supplemented with erythromycin or chloramphenicol $\left(10 \mu \mathrm{g} \mathrm{ml}^{-1}\right)$ as required. E. coli strains were cultivated in/on Luria-Bertani (LB) broth/agar supplemented with ampicillin $\left(100 \mu \mathrm{g} \mathrm{ml}^{-1}\right)$ as required.

\section{DNA manipulations and genetic techniques}

Genomic and plasmid DNA were isolated as previously described [7]. PCR assays to determine the presence of sssF (primers 1127 and 1128) were performed using Taq DNA polymerase (NEB) under the following conditions: 2 min at $94^{\circ} \mathrm{C}, 25$ cycles of $15 \mathrm{~s}$ at $94^{\circ} \mathrm{C}, 30 \mathrm{~s}$ at $55^{\circ} \mathrm{C}, 20 \mathrm{~s}$ at $72^{\circ} \mathrm{C}, 1$ cycle of $3 \mathrm{~min}$ at $72^{\circ} \mathrm{C}, 4^{\circ} \mathrm{C}$ hold. Primers were synthesised by Sigma and are listed in Table 2. PCR amplification of the $s s s F$ gene was performed using Phusion Hot Start DNA Polymerase (Finnzymes).

\section{Bioinformatic analysis and identification of $s s s F$}

The $s s s F$ gene was identified in plasmid pSSAP2 of S. saprophyticus MS1146. The final pSSAP2 sequence was finished to Q40 standard with an average Sanger read depth of $\sim 23 \times$ coverage, which corresponds to an estimated number of four pSSAP2 plasmid copies per cell, based on the observed chromosomal read coverage (data not shown). Annotation of plasmid pSSAP2 was carried out manually using Artemis [55] and BLAST [56] similarity searches of publicly available sequence databases. The complete nucleotide sequence of $S$. saprophyticus plasmid pSSAP2 is available from the GenBank/EMBL/DDBJ database under accession number HE616681. The multiple alignment (Additional file 2: Figure S1) was created with CLUSTAL W2 [57] and edited with Jalview [58]. Figure 1 was produced using Easyfig [59].

\section{Construction and complementation of staphylococcal mutants}

Plasmid construct pNK24 (Table 1), specifically retargeted to the $s s s F$ gene of $S$. saprophyticus MS1146, was prepared using the Sigma TargeTron Gene Knockout System, as per the manufacturer's instructions. Retargeting PCR primer sequences (1001-1003, Table 2) were determined by the TargeTron online design site, followed by a retargeting PCR and cloning of the PCR product into the provided shuttle vector, pNL9164 (Table 1). The construct was sequenced to verify correct inserts using primer 1011 (Table 2). The retargeted plasmid was then purified with a Qiagen Maxiprep kit and introduced into S. saprophyticus MS1146 by protoplast transformation as previously described [10], followed by $\mathrm{CdCl}_{2}$ induction and colony PCR screening to identify the sss $F$ mutant (MS1146sssF). The S. aureus SH1000 sasF gene was also interrupted with the TargeTron system as above, using primers 2065-2067 (Table 2). The retargeted plasmid (pNK41, Table 1) was passaged through a restrictiondeficient $S$. aureus strain (RN4220), then electroporated into S. aureus SH1000 and induced to create the sasF mutant (SH1000sasF). For complementation of the S. saprophyticus MS1146 sssF mutation, the sssF gene was initially amplified from S. saprophyticus MS1146 (primers 839 and 840, Table 2) and cloned into the BamHI site of pSK5632, forming plasmid pSKSssF. Plasmid pPS44 was digested with $\mathrm{BamHI} / \mathrm{XbaI}$ and the vector part was ligated with the BamHI/XbaI sssF-containing fragment from pSKSssF to generate plasmid pSssF. Plasmid pSssF was used to transform S. carnosus TM300, re-isolated and then introduced into $S$. saprophyticus MS1146sssF by protoplast transformation. For complementation of the SH1000sasF mutation, sasF from S. aureus SH1000 was PCR amplified (primers 2084 and 2085, Table 2) and cloned into the HindIII site of pSK5632 to form plasmid pSKSasF, followed by electroporation of SH1000sasF. SH1000sasF was heterologously complemented with the $S$. saprophyticus MS1146 sssF gene by the introduction of pSKSssF. S. aureus SH1000sasF containing empty pSK5632 vector was also prepared as a control.

\section{Purification of truncated SssF, antibody production and immunoblotting}

For antiserum production, a 1330 bp segment from sss $F$ from S. saprophyticus MS1146 (Figure 2A) was amplified with primers 873 and 874 (Table 2), digested with XhoI/ EcoRI and ligated into Xhol/EcoRI-digested pBAD/HisB. The resultant plasmid (pSssFHis) contained the base pairs 181-1510 of $s s s F$ fused to a $6 \times$ His-encoding sequence. This $s s S F$ sequence corresponds to amino residues 39-481 of the SssF sequence. Protein induction and 
Table 1 Strains and plasmids used in this study

\begin{tabular}{|c|c|c|}
\hline $\begin{array}{l}\text { Strain or } \\
\text { plasmid }\end{array}$ & Description & $\begin{array}{l}\text { Reference or } \\
\text { source }\end{array}$ \\
\hline \multicolumn{3}{|l|}{ E. coli strains } \\
\hline $\mathrm{DH} 5 \alpha$ & F- $\phi 80 \mathrm{~d} / a c Z \Delta \mathrm{M} 15 \Delta\left(\right.$ lacZYA-argF)U169 deoR recA1 endA1 hsdR17( $\left.\mathrm{r}_{\mathrm{k}}-\mathrm{m}_{\mathrm{k}}+\right)$ phoA supE44 $\lambda$ - thi-1 gyrA96 relA1 & Grant et al. [50] \\
\hline BL21 & F- ompT hsd $S_{B}\left(r_{B^{-}}-m_{B^{-}}\right)$gal dcm & Stratagene \\
\hline MS2066 & DH5 $\alpha$ containing pSssFHis & This study \\
\hline MS2067 & BL21 containing pSssFHis & This study \\
\hline \multicolumn{3}{|l|}{$\begin{array}{l}\text { S. saprophyticus } \\
\text { strains }\end{array}$} \\
\hline ATCC 15305 & Type strain (genome sequenced) & Kuroda et al. [8] \\
\hline MS1146 & Clinical isolate & AstraZeneca \\
\hline MS1146ssSF & MS1146 isogenic sssF mutant & This study \\
\hline MS1146sssF(pSssF) & Complemented MS1146 sssF mutant & This study \\
\hline \multicolumn{3}{|l|}{ S. aureus strains } \\
\hline SH1000 & Functional rsbU-repaired derivative of S. aureus 8325-4 & $\begin{array}{l}\text { Horsburgh et al. } \\
\text { [51] }\end{array}$ \\
\hline SH1000sasF & $\mathrm{SH} 1000$ isogenic sasF mutant & This study \\
\hline $\begin{array}{l}\text { SH1000sasF } \\
\text { (pSKSasF) }\end{array}$ & SH1000 sasF mutant complemented with sasF & This study \\
\hline $\begin{array}{l}\text { SH1000sasF } \\
\text { (pSKSssF) }\end{array}$ & SH1000 sasF mutant complemented with sssF & This study \\
\hline $\begin{array}{l}\text { SH1000sasF } \\
\text { (pSK5632) }\end{array}$ & SH1000 sasF mutant with empty pSK5632 vector & This study \\
\hline \multicolumn{3}{|l|}{$\begin{array}{l}\text { S. carnosus } \\
\text { strains }\end{array}$} \\
\hline TM300 & Wild-type SK311 & $\begin{array}{l}\text { Schleifer \& } \\
\text { Fischer [52] }\end{array}$ \\
\hline TM300(pSssF) & TM300 containing pSssF & This study \\
\hline \multicolumn{3}{|l|}{ Plasmids } \\
\hline $\mathrm{pBAD} / \mathrm{HisB}$ & Cloning and protein expression vector, containing $\mathrm{N}$-terminal $6 \times$ His tag; $\mathrm{Ap}^{r}$ & Invitrogen \\
\hline pNL9164 & E. coli/S. aureus TargeTron shuttle vector (temperature sensitive); $\mathrm{Ap}^{\mathrm{r}} \mathrm{Em}^{\mathrm{r}}$ & Sigma \\
\hline pSK5632 & Cloning and expression E. coli/S. aureus shuttle vector; $\mathrm{Ap}^{r} \mathrm{Cm}^{r}$ & $\begin{array}{l}\text { Grkovic et al. } \\
\text { [53] }\end{array}$ \\
\hline pPS44 & Staphylococcal vector, contains replicon and cat gene of $\mathrm{pC} 194 ; \mathrm{Cm}^{\mathrm{r}}$ & Wieland [54] \\
\hline pSssFHis & $\begin{array}{l}1330 \mathrm{bp} \text { MS1146 sssF fragment, amplified with primers } 873 \text { and 874, digested with EcoRI/Xhol and cloned } \\
\text { into EcoRI/Xhol-digested pBAD/HisB, with in-frame N-terminal } 6 \times \text { His tag; } \mathrm{Ap}^{r}\end{array}$ & This study \\
\hline pNK24 & $\begin{array}{l}\text { pNL9164 shuttle vector retargeted with primers 1001-1003, EBSU to knock out MS1146 sssF (TargeTron } \\
\text { system); } \text { Ap }^{r} \text { Em}^{r}\end{array}$ & This study \\
\hline pNK41 & $\begin{array}{l}\text { pNL9164 shuttle vector retargeted with primers 2065-2067, EBSU to knock out SH1000 sasF (TargeTron } \\
\text { system); } \text { Ap }^{r} \text { Em}^{r}\end{array}$ & This study \\
\hline pSKSssF & $\begin{array}{l}2394 \text { bp fragment, including entire sssF gene from MS1146, amplified with primers } 839 \text { and } 840 \text { and cloned } \\
\text { into the BamHI site of pSK5632; } \mathrm{Ap}^{r} \mathrm{Cm}^{r}\end{array}$ & This study \\
\hline pSssF & $\begin{array}{l}2400 \text { bp BamHI/Xbal fragment, containing sssF gene, subcloned from pSKSssF into BamHI/Xbal-digested } \\
\text { pPS44; } \mathrm{Cm}^{r}\end{array}$ & This study \\
\hline pSKSasF & $\begin{array}{l}2175 \text { bp fragment, including sasF gene from S. aureus NCTC 8325, amplified with primers } 2084 \text { and } 2085 \text { and } \\
\text { cloned into the Hindlll site of pSK5632; } \mathrm{Ap}^{r} \mathrm{Cm}^{r}\end{array}$ & This study \\
\hline
\end{tabular}

purification, inoculation of rabbits, staphylococcal cell lysate preparation and immunoblotting were performed as described previously [7], except NuPAGE Novex 4$12 \%$ Bis-Tris precast gels with NuPAGE MES SDS running buffer (Invitrogen) were used for the SDS-PAGE and S. saprophyticus MS1146sssF-adsorbed rabbit anti-
SssF serum was used as the primary serum for the Western blot.

Microscopy and image analysis

Immunogold labeling and transmission electron microscopy (TEM) were performed as described previously 
Table 2 PCR primers used in this study

\begin{tabular}{|c|c|c|}
\hline Primer & Sequence $\left(5^{\prime}-3^{\prime}\right)$ & Description \\
\hline 1127 & GTTGAAGCAATATTGAAGAAAGC & sssF screen forward \\
\hline 1128 & TTCTTCATTTAGTTTACCCATATCAAC & sssF screen reverse \\
\hline 839 & GCTAGGATCCTCCATCTAATTCAAATGACAACG & sssF cloning forward. Contains BamHI site (underlined) \\
\hline 840 & ACTAGGATCCGCTCCATTCAAAGTTCCACTTAC & sssF cloning reverse. Contains BamHI site (underlined) \\
\hline 873 & GCTCACTCGAGTTCGACACCATCAGTAGAAGC & $\begin{array}{l}\text { SSSF fragment PCR for cloning into } \mathrm{pBAD} / \mathrm{HisB} \text {, for } \\
\text { antibody production, forward. Contains Xhol site } \\
\text { (underlined) }\end{array}$ \\
\hline 874 & GCTCGGAATTCAAGCGCTITAGCTITAGCATC & $\begin{array}{l}\text { SSSF fragment PCR for cloning into } \mathrm{pBAD} / \mathrm{HisB} \text {, for } \\
\text { antibody production, reverse. Contains EcoRl site } \\
\text { (underlined) }\end{array}$ \\
\hline 1001 & AAAAAAGCTTATAATTATCCTTAAGTCACTACTATGTGCGCCCAGATAGGGTG & sssF TargeTron IBS \\
\hline 1002 & CAGATTGTACAAATGTGGTGATAACAGATAAGTCTACTATCTTAACTTACCTITCTTTGT & sssF TargeTron EBS1d \\
\hline 1003 & TGAACGCAAGTTTCTAATTTCGATTTGACTTCGATAGAGGAAAGTGTCT & sssF TargeTron EBS2 \\
\hline 2065 & AAAAAAGCTTATAATTATCCTTATCGTACGGCAAGGTGCGCCCAGATAGGGTG & sasF TargeTron IBS \\
\hline 2066 & CAGATTGTACAAATGTGGTGATAACAGATAAGTCGGCAAGATTAACTTACCTTTCTITGT & sasF TargeTron EBS1d \\
\hline 2067 & TGAACGCAAGTTTCTAATTTCGGTTTACGATCGATAGAGGAAAGTGTCT & sasF TargeTron EBS2 \\
\hline 2084 & CAGTAAGCTITGTTAGCGACATGGACAATATG & sasF cloning forward. Contains Hindlll site (underlined) \\
\hline 2085 & CCGTAAGCTITTGCATATACTTCACAATAAATTAAGG & sasF cloning reverse. Contains Hindlll site (underlined) \\
\hline 1011 & TTCTITAGGTGATGAACATATCAGG & $\begin{array}{l}\text { Sequencing primer to check for correct } 350 \mathrm{bp} \\
\text { retargeted intron fragments for TargeTron }\end{array}$ \\
\hline EBSU & CGAAATTAGAAACTTGCGTTCAGTAAAC & TargeTron EBS universal \\
\hline
\end{tabular}

[7], using 1:10 anti-SssF serum as the primary antibody. No negative staining was performed.

\section{Linoleic acid survival assay}

S. aureus linoleic acid survival assays were performed essentially as described by Kenny et al. [30]. Briefly, serial dilutions of overnight cultures $(2.5 \mu \mathrm{l}$ spots) were plated in duplicate onto $\mathrm{BHI}$ agar, $\mathrm{pH}$ 6.0, containing 0 $\mathrm{mM}$ or $1 \mathrm{mM}$ linoleic acid. All agar media contained a final concentration of $1 \%$ ethanol. Colonies were counted after overnight incubation at $37^{\circ} \mathrm{C}$. Mean values were compared using Student's $t$ test. S. saprophyticus survival assays were performed similarly, but with agar plates containing $5 \mathrm{mM}$ linoleic acid, supplemented with $0.85 \mathrm{M} \mathrm{NaCl}$.

\section{Structural predictions of SssF}

Secondary structure and three-dimensional fold predictions were performed using PSI-PRED [24] and Phyre [25], respectively.

\section{Additional material}

Additional file 1: Table S1. Predicted protein-coding genes of pSSAP2. Additional file 2: Figure S1. ClustalW alignment of the C-terminal 402 amino acid residues of S. saprophyticus MS1146 SssF protein (61\% of entire sequence) with corresponding sequence from other staphylococcal SssF-like proteins, showing clusters of amino acid conservation. Only one representative protein from each species is shown. Sequences are sorted (in descending order) by similarity to $S$. saprophyticus MS1146 SssF sequence, which ranges from 31.1\% (S. pseudintermedius HKU10-03) to $48.5 \%$ (S. carnosus TM300). Jalview was used to colour-code the alignment by percentage identity. The Cterminal sortase anchor motifs are indicated by a red box. GenBank accessions for the SssF-like proteins are as follows: S. carnosus TM300, CAL29334; S. capitis SK14, EEE48467; S. caprae C87, EFS16450; S. epidermidis RP62A, AAW53125; S. warneri L37603, EEQ79103; S. haemolyticus JCSC1435, BAE03665; S. hominis SK119, EEK11979; S. aureus NCTC 8325, ABD31969; S. lugdunensis HKU09-01, ADC86449; S. pseudintermedius HKU10-03, ADV06726.

\section{Acknowledgements}

This work was supported by grants from the Australian National Health and Medical Research Council to M.A.S. (569676) and S.A.B. (511224), and a University of Queensland Early Career Researcher grant to S.A.B. M.A.S. is supported by an Australian Research Council (ARC) Future Fellowship (FT100100662) and S.A.B. is supported by an ARC Australian Research Fellowship (DP0881247).

\section{Author details}

${ }^{1}$ Australian Infectious Diseases Research Centre, School of Chemistry and Molecular Biosciences, The University of Queensland, Brisbane, QLD 4072, Australia. ${ }^{2}$ Department of Medical Microbiology, Institute for Hygiene and Microbiology, Ruhr-Universität Bochum, Universitätsstr. 150, D-44780 Bochum, Germany. ${ }^{3}$ Institute for Molecular Bioscience, The University of Queensland, Brisbane, QLD 4072, Australia. ${ }^{4}$ Center for Infectious Diseases and Travel Medicine, University Medical Center Freiburg, Freiburg, Germany. ${ }^{5}$ School of Biosciences, University of Kent, Canterbury, Kent CT2 7NJ, UK.

\section{Authors' contributions}

NPK identified the SssF gene, participated in the design of the study, performed sequence analysis, performed the preliminary SssF phenotypic experiments, performed the PCR prevalence screening, prepared the sSSF antigen for antibody production, constructed the knockout mutants, performed the Western blots, prepared the samples for electron microscopy, 
performed the survival assays, and was the principal writer of the manuscript. TS performed the subcloning and transformations of $S$. saprophyticus and S. carnosus for the complementation of the $S$. saprophyticus MS1146 SSSF mutant, and assisted in editing the manuscript. NLBZ prepared Figure 1 and Additional file 1: Table S1 and assisted in writing and editing the manuscript. MT performed the electron microscopy and assisted in editing the manuscript. BH performed the structural predictions of SsSF and prepared Figure $2 \mathrm{~B}$ and $2 \mathrm{C}$. PS participated in the RP-HPLC and assisted in editing the manuscript. MS participated in the RPHPLC and assisted in editing the manuscript. SGG provided the German sssF prevalence data and assisted in editing the manuscript. SAB co-directed the research and assisted in writing and editing the manuscript. MAS directed the research and assisted in writing and editing the manuscript. All authors read and approved the final manuscript.

Received: 3 October 2011 Accepted: 15 January 2012 Published: 15 January 2012

\section{References}

1. Schappert SM: Ambulatory care visits to physician offices, hospital outpatient departments, and emergency departments: United States, 1997. Vital Health Stat 1999, 13(143):1-36.

2. Foxman B, Barlow R, D'Arcy H, Gillespie B, Sobel JD: Urinary tract infection: self reported incidence and associated costs. Ann Epidemiol 2000, 10(8):509-515.

3. Hooton TM, Stamm WE: Diagnosis and treatment of uncomplicated urinary tract infection. Infect Dis Clin North Am 1997, 11(3):551-581.

4. Rupp ME, Soper DE, Archer GL: Colonization of the female genital tract with Staphylococcus saprophyticus. J Clin Microbiol 1992, 30(11):2975-2979.

5. Rupp ME, Archer GL: Coagulase-negative staphylococci - pathogens associated with medical progress. Clin Infect Dis 1994, 19(2):231-243.

6. Faro S, Fenner DE: Urinary tract infections. Clin Obstet Gynecol 1998, 41(3):744-754.

7. King NP, Beatson SA, Totsika M, Ulett GC, Alm RA, Manning PA, Schembri MA: UafB is a serine-rich repeat adhesin of Staphylococcus saprophyticus that mediates binding to fibronectin, fibrinogen and human uroepithelial cells. Microbiology 2011, 157:1161-1175.

8. Kuroda M, Yamashita A, Hirakawa H, Kumano M, Morikawa K, Higashide M, Maruyama A, Inose Y, Matoba K, Toh H, et al: Whole genome sequence of Staphylococcus saprophyticus reveals the pathogenesis of uncomplicated urinary tract infection. Proc Natl Acad Sci USA 2005, 102(37):13272-13277.

9. Sakinç T, Kleine B, Gatermann SG: Sdrl, a serine-aspartate repeat protein identified in Staphylococcus saprophyticus strain 7108, is a collagenbinding protein. Infect Immun 2006, 74(8):4615-4623.

10. Hell W, Meyer HGW, Gatermann SG: Cloning of aas, a gene encoding a Staphylococcus saprophyticus surface protein with adhesive and autolytic properties. Mol Microbiol 1998, 29(3):871-881.

11. Meyer HGW, WenglerBecker U, Gatermann SG: The hemagglutinin of Staphylococcus saprophyticus is a major adhesin for uroepithelial cells. Infect Immun 1996, 64(9):3893-3896.

12. Sakinç T, Woznowski M, Ebsen M, Gatermann SG: The surface-associated protein of Staphylococcus saprophyticus is a lipase. Infect Immun 2005, 73(10):6419-6428.

13. Gatermann S, Marre R: Cloning and expression of Staphylococcus saprophyticus urease gene sequences in Staphylococcus carnosus and contribution of the enzyme to virulence. Infect Immun 1989, 57(10):2998-3002.

14. Schneider PF, Riley TV: Cell-surface hydrophobicity of Staphylococcus saprophyticus. Epidemiol Infect 1991, 106(1):71-75.

15. Atmaca S, Elci S, Akpolat NO: Differential production of slime by Staphylococcus saprophyticus under aerobic and anaerobic conditions. $J$ Med Microbiol 2000, 49(11):1051-1052.

16. Sakinç T, Michalski N, Kleine B, Gatermann SG: The uropathogenic species Staphylococcus saprophyticus tolerates a high concentration of D-serine. FEMS Microbiol Lett 2009, 299(1):60-64.

17. Colleen S, Hovelius B, Wieslander A, Mårdh PA: Surface properties of Staphylococcus saprophyticus and Staphylococcus epidermidis as studied by adherence tests and 2-polymer, aqueous phase systems. Acta Pathol Microbiol Scand [B] 1979, 87(6):321-328.

18. Hovelius B, Mårdh PA: Staphylococcus saprophyticus as a common cause of urinary tract infections. Rev Infect Dis 1984, 6(3):328-337.
19. Raz R, Colodner R, Kunin CM: Who are you - Staphylococcus saprophyticus? Clin Infect Dis 2005, 40(6):896-898.

20. Pead L, Maskell R: Micrococci and urinary infection. Lancet 1977, 2(8037):565-565.

21. Ito T, Katayama $Y$, Hiramatsu K: Cloning and nucleotide sequence determination of the entire mec DNA of pre-methicillin-resistant Staphylococcus aureus N315. Antimicrob Agents Chemother 1999, 43(6):1449-1458.

22. McKenzie T, Hoshino T, Tanaka T, Sueoka N: The nucleotide sequence of pUB110 - some salient features in relation to replication and its regulation. Plasmid 1986, 15(2):93-103.

23. Bendtsen JD, Nielsen $H$, von Heijne G, Brunak S: Improved prediction of signal peptides: SignalP 3.0. J Mol Biol 2004, 340:783-795.

24. Jones DT: Protein secondary structure prediction based on positionspecific scoring matrices. J Mol Biol 1999, 292(2):195-202.

25. Kelley LA, Sternberg MJE: Protein structure prediction on the Web: a case study using the Phyre server. Nat Protoc 2009, 4(3):363-371.

26. Murzin AG, Brenner SE, Hubbard T, Chothia C: SCOP: a structural classification of proteins database for the investigation of sequences and structures. J Mol Biol 1995, 247(4):536-540.

27. Berman HM, Westbrook J, Feng Z, Gilliland G, Bhat TN, Weissig H, Shindyalov IN, Bourne PE: The Protein Data Bank. Nucleic Acids Res 2000, 28(1):235-242.

28. Whitby FG, Phillips GN Jr: Crystal structure of tropomyosin at 7 Angstroms resolution. Proteins 2000, 38(1):49-59.

29. Ylanne J, Scheffzek K, Young P, Saraste M: Crystal structure of the alphaactinin rod reveals an extensive torsional twist. Structure 2001, 9(7):597-604.

30. Kenny JG, Ward D, Josefsson E, Jonsson IM, Hinds J, Rees HH, Lindsay JA, Tarkowski A, Horsburgh MJ: The Staphylococcus aureus response to unsaturated long chain free fatty acids: survival mechanisms and virulence implications. PLOS ONE 2009, 4(2):e4344.

31. Sakinç T, Kleine B, Michalski N, Kaase M, Gatermann SG: Sdrl of Staphylococcus saprophyticus is a multifunctional protein: localization of the fibronectin-binding site. FEMS Microbiol Lett 2009, 301(1):28-34

32. Navarre WW, Schneewind O: Surface proteins of Gram-positive bacteria and mechanisms of their targeting to the cell wall envelope. Microbiol Mol Biol Rev 1999, 63(1):174-229.

33. Roche FM, Massey R, Peacock SJ, Day NPJ, Visai L, Speziale P, Lam A, Pallen M, Foster TJ: Characterization of novel LPXTG-containing proteins of Staphylococcus aureus identified from genome sequences. Microbiology 2003, 149:643-654.

34. Long JP, Hart J, Albers W, Kapral FA: The production of fatty acid modifying enzyme (FAME) and lipase by various staphylococcal species. J Med Microbiol 1992, 37(4):232-234.

35. Mortensen JE, Shryock TR, Kapral FA: Modification of bactericidal fatty acids by an enzyme of Staphylococcus aureus. J Med Microbiol 1992, 36(4):293-298.

36. Chamberlain NR, Brueggemann SA: Characterisation and expression of fatty acid modifying enzyme produced by Staphylococcus epidermidis. J Med Microbiol 1997, 46(8):693-697.

37. Medical physiology: a cellular and molecular approach. Edited by: Boron WF, Boulpaep EL. Philadelphia, PA: Saunders/Elsevier; , 2 2009:

38. Kohler T, Weidenmaier C, Peschel A: Wall teichoic acid protects Staphylococcus aureus against antimicrobial fatty acids from human skin. J Bacteriol 2009, 191(13):4482-4484.

39. Clarke SR, Mohamed R, Bian L, Routh AF, Kokai-Kun JF, Mond Jر, Tarkowski A, Foster SJ: The Staphylococcus aureus surface protein IsdA mediates resistance to innate defenses of human skin. Cell Host Microbe 2007, 1(3):199-212.

40. Volkov A, Liavonchanka A, Kamneva O, Fiedler T, Goebel C, Kreikemeyer B, Feussner I: Myosin cross-reactive antigen of Streptococcus pyogenes M49 encodes a fatty acid double bond hydratase that plays a role in oleic acid detoxification and bacterial virulence. J Biol Chem 2010, 285(14):10353-10361.

41. Rosberg-Cody E, Liavonchanka A, Gobel C, Ross RP, O'Sullivan O, Fitzgerald GF, Feussner I, Stanton C: Myosin-cross-reactive antigen (MCRA) protein from Bifidobacterium breve is a FAD-dependent fatty acid hydratase which has a function in stress protection. BMC Biochem 2011, 12(9) 
42. Arpigny JL, Jaeger KE: Bacterial lipolytic enzymes: classification and properties. Biochem J 1999, 343:177-183.

43. Storch J, McDermott L: Structural and functional analysis of fatty acidbinding proteins. J Lipid Res 2009, 50:S126-S131.

44. Ricketts CR, Squire JR, Topley E, Lilly HA: Human skin lipids with particular reference to the self-sterilising power of the skin. Clin Sci 1951, 10(1):89-111.

45. Dye ES, Kapral FA: Survival of Staphylococcus aureus in intraperitoneal abscesses. J Med Microbiol 1981, 14(2):185-194.

46. Chapkin RS, Ziboh VA, Marcelo CL, Voorhees JJ: Metabolism of essential fatty acids by human epidermal enzyme preparations - evidence of chain elongation. J Lipid Res 1986, 27(9):945-954.

47. Huggins $G R$, Preti G: Volatile constituents of human vaginal secretions. Am J Obstet Gynecol 1976, 126(1):129-136.

48. Rankin DJ, Rocha EPC, Brown SP: What traits are carried on mobile genetic elements, and why? Heredity 2011, 106(1):1-10.

49. Eberhard WG: Why do bacterial plasmids carry some genes and not others? Plasmid 1989, 21(3):167-174.

50. Grant SGN, Jessee J, Bloom FR, Hanahan D: Differential plasmid rescue from transgenic mouse DNAs into Escherichia coli methylation-restriction mutants. Proc Natl Acad Sci USA 1990, 87(12):4645-4649.

51. Horsburgh MJ, Aish JL, White IJ, Shaw L, Lithgow JK, Foster SJ: Sigma(B) modulates virulence determinant expression and stress resistance: characterization of a functional $r s b U$ strain derived from Staphylococcus aureus 8325-4. J Bacterio/ 2002, 184(19):5457-5467.

52. Schleifer KH, Fischer U: Description of a new species of the genus Staphylococcus - Staphylococcus carnosus. Int I Syst Bacteriol 1982, 32(2):153-156.

53. Grkovic S, Brown MH, Hardie KM, Firth N, Skurray RA: Stable low-copynumber Staphylococcus aureus shuttle vectors. Microbiology 2003, 149:785-794.

54. Wieland B: Der Xyl-Promotor aus Staphylococcus xylosus als Grundlage der transtriptionale Regulation von Genen in Staphylococcus carnosus, PhD thesis. PhD thesis Tübingen, Germany: Universität Tübingen; 1993.

55. Rutherford K, Parkhill J, Crook J, Horsnell T, Rice P, Rajandream MA, Barrell B: Artemis: sequence visualization and annotation. Bioinformatics 2000, 16(10):944-945.

56. Altschul SF, Madden TL, Schaffer AA, Zhang JH, Zhang Z, Miller W, Lipman DJ: Gapped BLAST and PSI-BLAST: a new generation of protein database search programs. Nucleic Acids Res 1997, 25(17):3389-3402.

57. Chenna R, Sugawara H, Koike T, Lopez R, Gibson TJ, Higgins DG, Thompson JD: Multiple sequence alignment with the Clustal series of programs. Nucleic Acids Res 2003, 31(13):3497-3500.

58. Waterhouse AM, Procter JB, Martin DMA, Clamp M, Barton GJ: Jalview Version 2 - a multiple sequence alignment editor and analysis workbench. Bioinformatics 2009, 25(9):1189-1191.

59. Sullivan MJ, Petty NK, Beatson SA: Easyfig: a genome comparison visualiser. Bioinformatics (Oxf) 2011, doi: 10.1093/bioinformatics/btr039.

doi:10.1186/1471-2180-12-8

Cite this article as: King et al:: Characterisation of a cell wall-anchored protein of Staphylococcus saprophyticus associated with linoleic acid resistance. BMC Microbiology 2012 12:8.

\section{Submit your next manuscript to BioMed Central and take full advantage of:}

- Convenient online submission

- Thorough peer review

- No space constraints or color figure charges

- Immediate publication on acceptance

- Inclusion in PubMed, CAS, Scopus and Google Scholar

- Research which is freely available for redistribution

Submit your manuscript at www.biomedcentral.com/submit
C Biomed Central 